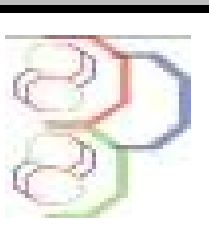

\title{
Évaluation des éléments traces métalliques dans les fientes de volaille recouvrant les coquilles d'œufs destinés à la consommation humaine en Côte d'Ivoire.
}

\author{
Apata Arsène ${ }^{1 *}$; Koffi Rose Nevrey ${ }^{1}$; Touré Nantarie2; Alui Konan Alphonse ${ }^{2}$ et Yao-Kouamé \\ Albert ${ }^{2}$ \\ 1, Université Nangui Abrogoua ; UFR des sciences et techniques des aliments. \\ 2, Université Félix Houphouët-Boigny de Cocody, UFR des Sciences de la Terre et des ressources Minières. \\ *= Auteur correspondant Email : apataarsen@yahoo.fr
}

Original submitted in on $17^{\text {th }}$ October 2013 Published online at www.m.elewa.org on $28^{\text {th }}$ February 2014.

http://dx.doi.org/10.4314/jab.v74i1.10

\section{RESUME}

Objectif: Mesurer le risque toxicologique dû aux éléments traces métalliques tels quel le plomb, le cadmium dans les fientes présentes sur les coquilles des œufs de poules pondeuses livrés sur les marchés de la ville d'Abidjan.

Méthodologie et résultats: Une enquête à été menée auprès de certains commerçants et consommateurs d'œufs. Les fientes présentes sur les coquilles de certains œufs livrés, le copeau de bois brut utilisé par les fermiers, l'aliment distribué aux volailles, ont été analysés selon la méthode ICPAES, au laboratoire LAPISEN de l'Institut National polytechnique de Yamoussoukro (INPHB). Les fientes prélevées après deux semaines et six mois d'élevage ont été également analysées selon la même méthode Les résultats des analyses en laboratoire réalisés sur le matériel biologique, indiquent, des teneurs moyennes en Fe de 289,67 mg. $\mathrm{kg}^{-1}$, dans la fiente, après deux semaines d'élevage, de $340,77 \mathrm{mg} \cdot \mathrm{kg}^{-1}$, dans la fiente, après six mois d'élevage, et de $844,46 \mathrm{mg} \cdot \mathrm{kg}^{-1}$, dans la fiente prélevée sur les coquilles d'œuf. La teneur moyenne en Cd est également de $7,81 \mathrm{mg} \cdot \mathrm{kg}^{-1}$ dans le copeau de bois, de $6,05 \mathrm{mg} \cdot \mathrm{kg}^{-1}$ dans les aliments, de $6,11 \mathrm{mg} \cdot \mathrm{kg}^{-1}$ dans la fiente, après deux semaines d'élevage, de 9,1 mg. $\mathrm{kg}^{-1}$ dans la fiente après six mois d'élevage et, en fin, de 9,26 mg. $\mathrm{kg}^{-1}$, dans la fiente recueillie directement sur les œufs des marchés faisant parties de notre étude. Les valeurs limitent en ces deux éléments, selon la norme CEE (Commission Économique des nations unies pour l'Europe), sont de $200 \mathrm{mg} \mathrm{kg}^{-1}$ pour le Fe et $1 \mathrm{mg} \cdot \mathrm{kg}^{-1}$ pour le $\mathrm{Cd}$.

Conclusion et application de résultats : les fientes présentes sur les coquilles de ces œufs contiennent après analyse des éléments traces métalliques. Cette présence d'éléments traces métalliques, surtout, non essentiels, montre le risque toxicologique, aux conséquences graves, auquel sont exposés les vendeurs et les consommateurs d'œufs à coquilles contaminées. II est urgent qu'une action de sensibilisation soit menée par le politique auprès des fabriques d'aliments de ces poules pondeuses afin que ces aliments soient dépourvus de plomb et de cadmium qui sont non essentiels pour l'organisme animal et toxique pour l'homme qui manipules les œufs à mains nues

Mots- clés : Éléments traces métalliques, fiente, coquilles d'œufs, Côte d'Ivoire. 


\section{ABSTRACT}

Objective: Evaluate the toxicological risk of trace metal from the droppings on chicken egg sold on the markets of Abidjan.

Methodology and results: An investigation was carried out with some traders and consumers of chicken eggs in Abidjan. The droppings on eggs shells, the woods shavings and the chicken feed used by the farmers, after two weeks and six months of breeding were analyzed for according ICP-AES method in the laboratory LAPISEN of Yamoussoukro. The content of iron was $289.67 \mathrm{mg} . \mathrm{kg}^{-1}, 340.77 \mathrm{mg}^{-\mathrm{kg}^{-1}}$ and $844.46 \mathrm{mg} \cdot \mathrm{kg}^{-1}$ respectively in the chicken wastes drawn after two weeks and six months of breeding and in the droppings on shells. The content of cadmium was $7.81 \mathrm{mg} \cdot \mathrm{kg}^{-1}$ in the shaving and 6.05 mg. $\mathrm{kg}^{-1}$ in the chicken feed. Those in the chicken wastes drawn after two weeks and six months of breeding and in the shell droppings were respectively $6.11 \mathrm{mg} \cdot \mathrm{kg}^{-1}, 9.1 \mathrm{mg} \cdot \mathrm{kg}^{-1}$ and $9.26 \mathrm{mg}^{-1} \mathrm{~kg}^{-1}$. However, the contents of $\mathrm{Fe}$ and $\mathrm{Cd}$ obtained in this study are higher than the $200 \mathrm{mg} \cdot \mathrm{kg}^{-1}$ for Fe and the $1 \mathrm{mg}^{\mathrm{kg}} \mathrm{k}^{-1}$ for $\mathrm{Cd}$ recommended by the CEE (Economic Commission of United Nations for Europe) as the limit values.

Conclusion and application of results: The analysis of the droppings on the shells of chicken eggs revealed the presence of trace metal elements, indicating the toxicological risk for sellers and consumers. Thus, a consciousness-raising is necessary by human to avoid the toxicity risk of the droppings on the chicken eggshell.

Keywords: Trace metals, droppings, eggshells, Côte d'Ivoire.

\section{INTRODUCTION}

Les éléments traces métalliques (ETM) ou métalloïdes sont des métaux dont la concentration n'excède pas $1000 \mathrm{mg} / \mathrm{kg}$ dans un échantillon ou un milieu donné (Basta et al., 2005). Ces ETM sont le manganèse ( $\mathrm{Mn})$, le fer $(\mathrm{Fe})$, le zinc $(\mathrm{Zn})$, le plomb $(\mathrm{Pb})$, le cuivre $(\mathrm{Cu})$, le cadmium (Cd), le nickel (Ni), le chrome ( $\mathrm{Cr}$ ) et l'arsenic (As) et le mercure (Hg). Les ETM, tels que $\mathrm{Mn}, \mathrm{Fe}, \mathrm{Zn}, \mathrm{Cu}, \mathrm{Ni}, \mathrm{Cr}$, et l'As sont des oligoéléments essentiels ou bénéfiques aux êtres vivants à certaines concentration. D'autres, comme le cadmium, le mercure et le plomb, sont des contaminants stricts, puisqu'ils n'ont pas d'utilité et sont donc toxiques pour les êtres vivants (Tremel-Schaub et Feix, 2005). Ces ETM peuvent se retrouver dans les plantes à partir $d u$ sol agricole du faite de six sources de contamination principales identifiées (traitements phytosanitaires, engrais minéraux, déjections animales, amendements calciques et magnésiens, boues et composts et retombées atmosphériques) ou des eaux polluées (ADEME, 2007). Et ces plantes sont utilisées pour l'alimentation des animaux, mais, aussi de l'homme. L'homme peut s'intoxiquer donc en consommant ces plantes directement ou par consommation d'animaux nourris avec ces plantes (SEMDE, 2005, Dauguet et al., 2011). Mais également en rentrant en contacte avec les déjections de ces animaux notamment les fientes de poules pondeuses. Les ETM peuvent, également, atteindre l'homme par passage à travers la peau (Gouzi et Ducos, 2008), par ingestion ou par inhalation (Benedetto et al, .1997). La manipulation, sans protection, des fientes de poules peut donc être source de contamination aux ETM pour l'homme. Le cassage des coquilles des œufs de poules pondeuses ayant de la fientes sur la surface sans nettoyage préalable peut être une voie de contamination de l'œuf et donc de celui qui le consomme. L'œuf est un aliment à grande consommation. Sa production mondiale était, en 2006, de 61,11 millions de tonnes (FAO, 2008). La production mondiale a augmentée $35 \%$ sur la décennie (Magdelaine et Braine, 2008). En Côte D'Ivoire, selon les données de la FAO (2008) la production avicole fournit entre 500 à 550 millions d'œufs par an. L'œuf est beaucoup consommé et il apporte à l'homme des protéines de très haute valeur biologique, capables de réparer et de fabriquer de nouveaux tissus. Les 
protéines de l'œuf sont si équilibrées qu'elles sont prises comme référence par la FAO pour juger de la qualité des autres sources de protéines (Faverger, 2005). Malgré sa richesse nutritive, l'œuf est beaucoup incriminé dans les toxi-infections alimentaires à Salmonella spp et Staphylococcus aureus (Tsen et al., 2000; Boisson et Teyssou, 2002; Anonyme 2; 2012). II représente un véritable problème de santé publique. Mis à part le facteur microbiologique, qui est toujours incriminé, il est important de surveiller le risque toxicologique

\section{MATERIEL ET METHODE}

Milieu d'étude : Abidjan, capitale économique de la Côte d'Ivoire, est la ville la plus peuplée de l'Afrique de l'Ouest francophonie. L'agglomération d'Abidjan est située au Sud de la Côte d'Ivoire et est comprise que pourrait représenter l'œuf livré sur le marché par les fermes du secteur informel, sans aucune précaution d'hygiène (FAO, 2008). C'est dans ce but que cette étude a été menée dans certaines communes de la ville d'Abidjan. Un questionnaire a été soumis, tant aux vendeurs qu'aux consommateurs d'œufs de poules. Par ailleurs, la quantification en ETM de fientes présentes sur les coquilles de certains œufs livrés sur les marchés des quartiers visités par les fermes du secteur informel à été réalisée au cours de cette étude.

entre les latitudes $5^{\circ} 00^{\prime}$ et $5^{\circ} 30^{\prime} \mathrm{N}$ et les longitudes $3^{\circ} 50^{\prime}$ et $4^{\circ} 10^{\prime} \mathrm{W}$ avec une superficie de 57.735 ha. (Anonyme, 2013).



Figure 1 : Localisation des différents sites de prélèvement (Touré, 2012). 
Matériel biologique: Fiente de poule: Selon Boughaba (2012), les fientes de poules sont constituées de fèces, d'urines, de plumes, d'œufs ou de coquilles d'œuf, et de litière. OEufs: les œufs

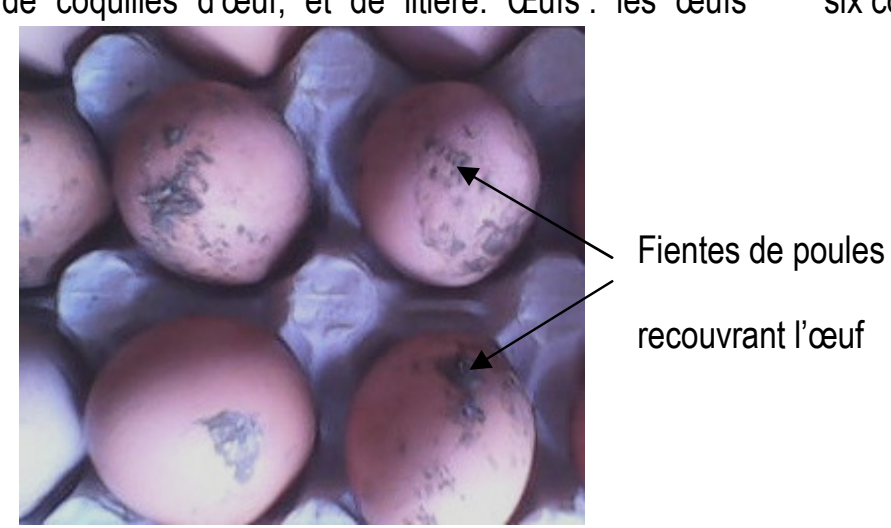

Figure 2 : Coquilles avec présence de fiente.

\section{Matériel de collecte d'information et de traitement des données}

$\checkmark \quad$ une fiche d'enquête comportant un questionnaire a été soumis tant aux vendeurs ainsi qu'aux consommateurs d'œufs de poules.

$\checkmark \quad$ les données obtenues ont été traitées à l'aide du logiciel STATISTICA 7.1

\section{Méthodes}

Échantillonnage: Six communes (Abobo, Adjamé, Yopougon, Cocody, Trechville, Koumassi) de la ville d'Abidjan ont été choisies car les œufs en provenance de l'intérieur du pays sont déversés directement sur leurs marchés. Les œufs ont été prélevés sur ces différents marchés en fonction de l'hygiène apportée, principalement, au niveau de la coquille. L'échantionnage a eu lieu sur une période de 6 mois, allant de Janvier 2012 à juin 2012. Le choix des œufs s'est effectué sans distinction de la provenance. Les échantillons de fientes ont été recueillis en grattant la coquille des œufs. Ainsi $500 \mathrm{~g}$ de fiente ont été prélevés par zone pour analyse. Les fermes industrielles livrant des œufs déjà nettoyés n'ont pas été considérées lors de cette étude. Les œufs ont été prélevés, de façon aléatoire, principalement ceux n'ayant subi aucun nettoyage par les vendeurs. Les marchés ont été visités de

\section{RÉSULTATS}

Age des participants: Les analyses statistiques appliquées au test relatif à l'âge des participants ayant été soumis à un questionnaire dont l'âge est supérieur à 25 ans comporte une différence hautement significative dans le cas des vendeurs et des consommateurs avec, des valeurs de $\mathrm{F}_{\text {cal }}=$ $817,14^{* *} ; P_{\text {cal }}=0.00012$. En effet, il ressort de cette étude que $62,65 \%$ des vendeurs on un âge prélevés au cours de cette étude étaient ceux livrés par les fermes du secteur informel sur les différents marchés de la ville d'Abidjan, en particulier, ceux des six communes visitées.

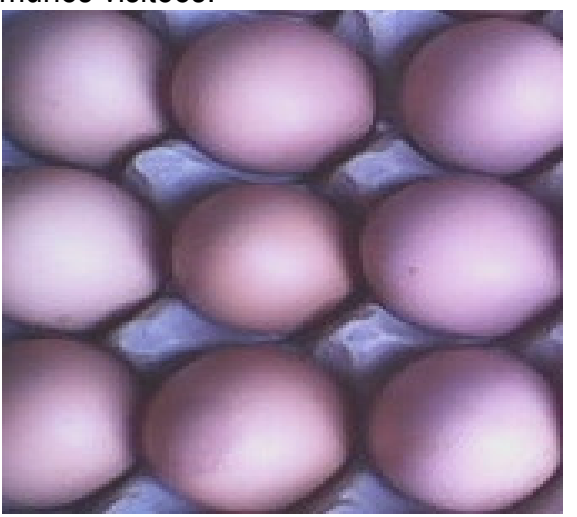

Figure 3 : CEufs propres.

façon aléatoire et un questionnaire leur a été soumis, tout comme à certains habitants volontaires dans chaque zone de prélèvement. Le questionnaire portait sur l'âge et le niveau de scolarisation. Au total, $500 \mathrm{~g}$ de copeaux de bois, d'aliment, de fiente de deux semaines et de six mois d'élevage, et sur les coquilles dans les marchés, ont été prélevés et conditionnés dans des sachets en polyéthylène. Chaque échantillon a subi, par la suite, un séchage, puis un tamisage. Des analyses de laboratoire ont été appliquées à chaque échantillon.

Analyse des échantillons: Afin de déterminer les teneurs en ces éléments traces métalliques, les échantillons prélevés ont été analysés par la méthode ICP-AES, au laboratoire LAPISEN de I'Institut National polytechnique de Yamoussoukro (INPHB).

Analyses statistiques des données : Les résultats obtenus ont été analysés statistiquement à l'aide du logiciel STATISTICA 7.1 par l'analyse de la variance (ANOVA). Chaque fois qu'une différence significative est révélée, l'ANOVA est complétée par le test de Tukey, qui permet d'identifier la (ou, les) variable(s) significativement différente(s) des autres. Les moyennes des variables ont été séparées au seuil a $=0,05$ et $\alpha=0,01$.

supérieur à 25 ans. Cette tranche d'âge a une proportion de $60,44 \%$ chez les consommateurs. Les participants d'âge compris entre 18 et 25 ans ont une proportion de $19,64 \%$ pour les vendeurs et $22,15 \%$ pour les consommateurs. Ceux ayant moins de 18 ans n'étaient pas négligeables car elle représente $16,64 \%$ dans le cas des vendeurs et $17,44 \%$ pour les consommateurs (Tableau1). 
Tableau 1 : Age relatif et niveau de scolarisation des participants à cette étude.

\begin{tabular}{|c|c|c|c|c|c|c|}
\hline & & \multicolumn{2}{|c|}{ Proportion } & \multirow{2}{*}{$F_{\text {cal }}$} & \multirow{2}{*}{$\mathbf{P}_{\text {cal }}$} & \multirow{2}{*}{$P_{\text {theo }}$} \\
\hline & & Vendeurs & Consommateurs & & & \\
\hline \multirow{3}{*}{ Tranche d'âge } & $<18$ & $16,64 \pm 0,02 b$ & $17,44 \pm 0,02 b$ & \multirow{3}{*}{$817,14^{* *}$} & \multirow{3}{*}{0.00012} & \multirow{3}{*}{$\leq 0,01$} \\
\hline & $18-25$ & $19,64 \pm 0,03 b$ & $22,15 \pm 0,02 b$ & & & \\
\hline & $>25$ & $62,65 \pm 0,02 \mathrm{a}$ & $60,44 \pm 0,03 a$ & & & \\
\hline \multirow{5}{*}{$\begin{array}{l}\text { Niveau de } \\
\text { scolarisation des } \\
\text { participants }\end{array}$} & Non lettré & \multicolumn{2}{|c|}{$9,84 \pm 1,02 c$} & & & \\
\hline & coranique & \multicolumn{2}{|c|}{$1,35 \pm 0,02 d$} & & & \\
\hline & primaire & \multicolumn{2}{|c|}{$24,364.8 b$} & $315,41^{* *}$ & 0,000231 & $\leq 0,01$ \\
\hline & secondaire & \multicolumn{2}{|c|}{$43,45 \pm 8.32 a$} & & & \\
\hline & universitaire & \multicolumn{2}{|c|}{$18,45 \pm 4.25 b$} & & & \\
\hline
\end{tabular}

Les moyennes affectées d'une même lettre dans une même colonne ne sont pas significativement différentes au seuil à < 0,01 selon le test de Tukey. ns $=$ non significative; ${ }^{*}=$ significative; ${ }^{* *}=$ hautement significative et ${ }^{* * *}=$ très hautement significative, $\mathrm{Cal}=$ calculer, théor $=$ théorique.

Niveau intellectuel des participants: Le niveau intellectuel au cours d'une enquête relatif à la consommation d'aliment pourrait être fondamental dans le cas où il annonce éventuellement une sensibilisation à réaliser. Les résultats de nos investigations, en ce qui concerne la proportion du niveau d'étude, comporte une différence hautement significative du point de vue statistique $\left(\mathrm{F}_{\mathrm{cal}}=\right.$ $315,41^{* *} ; P_{\text {cal }}=0,00341$ ). Ces résultats nous révèlent que $43,45 \%$ des participants ont un niveau du secondaire, suivi de $24,36 \%$ pour le primaire; $18,45 \%$ de ces participants ont un niveau d'enseignement supérieur et $1,35 \%$ ont fait seulement l'école coranique. II est à noter que $9,84 \%$ des enquêtés n'ont pas été scolarisés (Tableau 1).

Contenu en éléments trace métallique des fientes:La concentration en éléments traces métalliques des fientes dans les 6 communes visitées lors de ces travaux n'est pas significativement différente pour un même élément, d'une commune ou ville à une autre. Elle est par exemple, en moyenne de 30,$4 ; 844 ; 25,6 ; 8,3$;

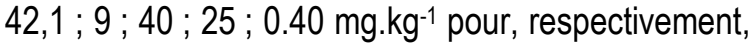
le manganèse $(\mathrm{Mn})$, le fer $(\mathrm{Fe})$, le zinc $(\mathrm{Zn})$, le plomb $(\mathrm{Pb})$, le cuivre $(\mathrm{Cu})$, le cadmium $(\mathrm{Cd})$, le nickel $(\mathrm{Ni})$, le chrome (Cr) et l'arsenic (As) (tableau 2\&3). Cependant ces charges en ETM sont significativement différentes d'un type d'échantillon à un autre et d'un élément métallique à un autre. C'est par exemple le cas zinc, avec une charge moyenne de $4 \mathrm{mg} . \mathrm{kg}^{-1}$ dans le copeau de bois, de $68 \mathrm{mg} \cdot \mathrm{kg}^{-1}$, dans la fiente, après 6 mois d'élevage, et de 2 mg. $\mathrm{kg}^{-1}$ de nickel, dans l'aliment de poule. Les éléments traces métalliques, considérés, par la CEE, ont des concentrations en deçà des valeurs seuil. Le cadmium est le seul ETM à valeurs supérieurs à la valeur seuil, quelques soit le type de substrat analysés (tableau 4). 
Tableau 2 : Éléments traces métalliques essentiels déterminés dans les différents substrats analysés.

\begin{tabular}{|c|c|c|c|c|c|c|c|c|}
\hline \multirow[b]{2}{*}{ Type de matériel } & \multirow[b]{2}{*}{ lieu de provenance } & \multicolumn{7}{|c|}{ Éléments traces métalliques essentiels (mg.kg-1) } \\
\hline & & $\mathrm{Mn}$ & $\mathrm{Fe}$ & $\mathrm{Zn}$ & $\mathrm{Cu}$ & $\mathrm{Ni}$ & $\mathrm{Cr}$ & As \\
\hline \multirow{6}{*}{ copeaux de bois } & Adzopé & $16,35 \pm 0,13 \mathrm{a}$ & $58,72 \pm 0,13 a$ & $4,75 \pm 0,06 a$ & $8,35 \pm 0,13 a$ & $0,27 \pm 0,1 b$ & $6,525 \pm 0,12 a$ & $0,44 \pm 0,02 a$ \\
\hline & Agnibilékro & $15,4 \pm 0,16 b$ & $57,76 \pm 0,11 b$ & $4,28 \pm 0,13 b$ & $7,78 \pm 0,13 b$ & $0,18 \pm 0,08 c$ & $6,42 \pm 0,08 a$ & $0,36 \pm 0,01 b$ \\
\hline & Azaguié & $14,46 \pm 0,23 c$ & $58,56 \pm 0,11 a$ & $4,78 \pm 0,08 a$ & $7,44 \pm 0,11 b$ & $0,46 \pm 0,11 a$ & $4,22 \pm 0,08 c$ & $0,43 \pm 0,02 a$ \\
\hline & Bingerville & $16,32 \pm 0,13 a$ & $57,24 \pm 0,11 b$ & $4,4 \pm 0,16 b$ & $8,42 \pm 0,13 a$ & $0,42 \pm 0,08 a$ & $5,4 \pm 0,15 b$ & $0,23 \pm 0,02 c$ \\
\hline & Dabou & $15,56 \pm 0,21 b$ & $58,66 \pm 0,15 a$ & $4,24 \pm 0,11 b$ & $7,6 \pm 0,16 b$ & $0,26 \pm 0,11 b$ & $5,22 \pm 0,13 b$ & $0,37 \pm 0,02 b$ \\
\hline & Divo & $16,38 \pm 0,13 a$ & $57,3 \pm 0,16 b$ & $3,7 \pm 0,16 \mathrm{c}$ & $7,66 \pm 0,18 b$ & $0,32 \pm 0,08 a b$ & $3,82 \pm 0,08$ & $0,43 \pm 0,02 a$ \\
\hline \multirow{6}{*}{ Aliments de poules } & Adzopé & $32,3 \pm 1,29 a$ & $139,62 \pm 0,17 b$ & $80,27 \pm 0,22 b$ & $21,17 \pm 0,10 a$ & $2,1 \pm 0,16 a$ & $9,5 \pm 0,08 a$ & $0,42 \pm 0,02 \mathrm{a}$ \\
\hline & Agnibilékro & $29,84 \pm 0,15 b$ & $139,76 \pm 0,09 a$ & $80,3 \pm 0,16 b$ & $21,06 \pm 0,09 b$ & $2,04 \pm 0,18 a$ & $9,5 \pm 0,07 a$ & $0,34 \pm 0,02 b$ \\
\hline & Azaguié & $29,82 \pm 0,04 b$ & $139,64 \pm 0,13 b$ & $80,38 \pm 0,15 b$ & $21,06 \pm 0,13 b$ & $2,1 \pm 0,15 a$ & $9,44 \pm 0,13 b$ & $0,43 \pm 0,02 a$ \\
\hline & Bingerville & $29,9 \pm 0,012 b$ & $139,74 \pm 0,09 b$ & $80,42 \pm 0,15 a$ & $21,22 \pm 0,13 a$ & $2,16 \pm 0,13 a$ & $9,44 \pm 0,09 b$ & $0,38 \pm 0,01 b$ \\
\hline & Dabou & $29,6 \pm 0,34 b$ & $139,68 \pm 0,04 b$ & $80,38 \pm 0,19 b$ & $21,12 \pm 0,13 b$ & $1,98 \pm 0,13 b$ & $9,52 \pm 0,04 a$ & $0,45 \pm 0,01 \mathrm{a}$ \\
\hline & Divo & $29,8 \pm 0,07 b$ & $139,64 \pm 0,09 b$ & $80,5 \pm 0,07 a$ & $21,1 \pm 0,14 b$ & $2,04 \pm 0,15 a$ & $9,56 \pm 0,13 a$ & $0,34 \pm 0,01 a$ \\
\hline \multirow{6}{*}{$\begin{array}{l}\text { Fiente après } 2 \text { semaines } \\
\text { d'élevage }\end{array}$} & Adzopé & $8,95 \pm 0,01 b$ & $297,84 \pm 0,02 a$ & $21,66 \pm 0,02 b$ & $9,35 \pm 0,24 a$ & $1,43 \pm 0,02 c$ & $7,45 \pm 0,03 a$ & $0,33 \pm 0,02 b$ \\
\hline & Agnibilékro & $7,74 \pm 0,02 c$ & $287,35 \pm 0,02 b$ & $20,96 \pm 0,02 c$ & $8,73 \pm 0,02 c$ & $1,74 \pm 0,02 b$ & $7,85 \pm 0,03 a$ & $0,276 \pm 0,01 c$ \\
\hline & Azaguié & $8,66 \pm 0,01 b$ & $294,16 \pm 0,03 a$ & $21,03 \pm 0,01 b$ & $9,52 \pm 0,02 a$ & $1,53 \pm 0,01 \mathrm{c}$ & $7,05 \pm 0,02 b$ & $0,37 \pm 0,01 b$ \\
\hline & Bingerville & $9,26 \pm 0,02 \mathrm{a}$ & $277,53 \pm 0,02 c$ & $22,64 \pm 0,02 \mathrm{a}$ & $8,77 \pm 0,02 b$ & $1,83 \pm 0,01 a$ & $6,95 \pm 0,03 c$ & $0,342 \pm 0,01 b$ \\
\hline & Dabou & $8,254 \pm 0,02 b$ & $285,34 \pm 0,02 b$ & $21,54 \pm 0,01 b$ & $9,03 \pm 0,01 a b$ & $1,34 \pm 0,02 \mathrm{c}$ & $7,84 \pm 0,02 a$ & $0,43 \pm 0,02 a$ \\
\hline & Divo & $8,23 \pm 0,02 b$ & $295,97 \pm 0,01 \mathrm{a}$ & $22,73 \pm 0,02 a$ & $8,924 \pm 0,02 c$ & $1,66 \pm 0,02 b$ & $7,42 \pm 0,01 \quad a$ & $0,33 \pm 0,02 b$ \\
\hline \multirow{6}{*}{$\begin{array}{l}\text { Fiente après } 6 \text { mois } \\
\text { d'élevage }\end{array}$} & Adzopé & $9,54 \pm 0,02 a$ & $339,65 \pm 0,021 b$ & $68,54 \pm 0,01 \mathrm{~b}$ & $26,24 \pm 0,01 \mathrm{~b}$ & $1,87 \pm 0,02 b$ & $9,54 \pm 0,02 \mathrm{a}$ & $0,54 \pm 0,02 \mathrm{a}$ \\
\hline & Agnibilékro & $8,96 \pm 0,02 b$ & $334,44 \pm 0,022 b$ & $68,15 \pm 0,04 b$ & $25,73 \pm 0,02 a$ & $1,75 \pm 0,02 c$ & $8,96 \pm 0,02 b$ & $0,96 \pm 0,02 b$ \\
\hline & Azaguié & $9,44 \pm 0,01 a$ & $341,35 \pm 0,023 a$ & $67,45 \pm 0,02 b$ & $26,83 \pm 0,02 b$ & $1,84 \pm 0,02 b$ & $9,44 \pm 0,01 a$ & $0,44 \pm 0,13 a$ \\
\hline & Bingerville & $9,23 \pm 0,01 a$ & $351,23 \pm 0,027 a$ & $70,15 \pm 0,03 a$ & $25,85 \pm 0,02 a$ & $1,96 \pm 0,05 a$ & $9,23 \pm 0,01 a$ & $0,23 \pm 0,01 a$ \\
\hline & Dabou & $8,87 \pm 0,02 b$ & $340,31 \pm 0,033 b$ & $69,54 \pm 0,02 \mathrm{a}$ & $26,03 \pm 0,02 b$ & $1,86 \pm 0,01 b$ & $8,87 \pm 0,02 b$ & $0,87 \pm 0,02 b$ \\
\hline & Divo & $8,95 \pm 0,02 c$ & $337,64 \pm 0,011 b$ & $68,42 \pm 0,02 b$ & $25,98 \pm 0,01 a$ & $1,85 \pm 0,01 b$ & $8,95 \pm 0,02 b$ & $0,95 \pm 0,01 b$ \\
\hline \multirow{6}{*}{$\begin{array}{l}\text { Fiente prélevée sur les } \\
\text { marchés des différentes } \\
\text { communes d'Abidjan }\end{array}$} & Abobo gare & $30,43 \pm 0,06 b$ & $844,12 \pm 0,02$ & $25,68 \pm 0,02$ & $42,15 \pm 0,01$ & $4,03 \pm 0,02$ & $2,53 \pm 0,02$ & $0,43 \pm 0,02$ \\
\hline & Adjamé marché & $30,44 \pm 0,04 a$ & $844,12 \pm 0,01$ & $25,67 \pm 0,02$ & $42,08 \pm 0,02$ & $4,02 \pm 0,03$ & $2,53 \pm 0,02$ & $0,4 \pm 0,02$ \\
\hline & yopougon siporex & $30,44 \pm 0,02 a$ & $844,18 \pm 0,02$ & $25,66 \pm 0,02$ & $42,07 \pm 0,02$ & $4,02 \pm 0,01$ & $2,53 \pm 0,01$ & $0,37 \pm 0,02$ \\
\hline & Cocody Angré marché cocovico & $30,43 \pm 0,01 b$ & $844,17 \pm 0,01$ & $25,67 \pm 0,02$ & $42,14 \pm 0,01$ & $4,02 \pm 0,02$ & $2,53 \pm 0,02$ & $0,43 \pm 0,02$ \\
\hline & Treichville marhé & $30,44 \pm 0,01 a$ & $844,97 \pm 0,45$ & $25,75 \pm 01,79$ & $42,06 \pm 0,02$ & $4,02 \pm 0,02$ & $2,51 \pm 0,47$ & $0,39 \pm 0,03$ \\
\hline & koumassi marché & $30,43 \pm 0,02 b$ & $845,18 \pm 0,02$ & $25,67 \pm 0,01$ & $42,11 \pm 0,01$ & $4,03 \pm 0,02$ & $2,54 \pm 0,02$ & $0,42 \pm 0,02$ \\
\hline Fcal & & $294,1652^{* *}$ & $501,4617^{\star *}$ & $649,5087^{* *}$ & $766,8259^{* *}$ & $3,2527^{*}$ & $0,331 \mathrm{~ns}$ & $115,8955^{\star *}$ \\
\hline Pcal & & 0,000558 & 0,000784 & 0,000311 & 0,000053 & 0,01073 & 0,564 & 0,000889 \\
\hline Pthéorique & & $\leq 0,01$ & $\leq 0,01$ & $\leq 0,01$ & $\leq 0,01$ & $\leq 0,05$ & $\geq 0,05$ & $\leq 0,01$ \\
\hline
\end{tabular}


Les moyennes affectées d'une même lettre dans une même colonne ne sont pas significativement différentes au seuil a $<0,01$ selon le test de Tukey. ns $=$ non significative; $\quad{ }^{*}=$ significative; ${ }^{* *}=$ hautement significative et ${ }^{* * *}=$ très hautement significative, Cal $=$ calculer, théor $=$ théorique.

Tableau 3 : Éléments traces métalliques non essentiels déterminés dans les différents substrats analysés.

\begin{tabular}{|c|c|c|c|}
\hline & & \multicolumn{2}{|c|}{ Eléments traces métalliques non essentiels (mg.kg-1) } \\
\hline Type de matériel & lieu de provenance & $\mathrm{Pb}$ & $\mathrm{Cd}$ \\
\hline \multirow{6}{*}{ copeaux de bois } & Adzopé & $1,25 \pm 0,13 a$ & $7,05 \pm 0,02 b$ \\
\hline & Agnibilékro & $1,24 \pm 0,11 a$ & $6,43 \pm 0,02 c$ \\
\hline & Azaguié & $1,16 \pm 0,05 a$ & $9,13 \pm 0,01 a$ \\
\hline & Bingerville & $0,8 \pm 0,1 \mathrm{~b}$ & $7,85 \pm 0,02 a$ \\
\hline & Dabou & $1,2 \pm 0,07 a$ & $8,66 \pm 0,02 a b$ \\
\hline & Divo & $1,18 \pm 0,08 a$ & $7,75 \pm 0,01 b$ \\
\hline \multirow{6}{*}{ Aliments de poules } & Adzopé & $10,12 \pm 0,13 b$ & $6,44 \pm 0,03 a$ \\
\hline & Agnibilékro & $10,14 \pm 0,05 b$ & $5,46 \pm 0,02 b$ \\
\hline & Azaguié & $10,28 \pm 0,25 a$ & $4,47 \pm 0,02 c$ \\
\hline & Bingerville & $10,08 \pm 0,04 b$ & $6,64 \pm 0,02 a$ \\
\hline & Dabou & $10,1 \pm 0,01 b$ & $6,76 \pm 0,02 a$ \\
\hline & Divo & $10,12 \pm 0,04 b$ & $6,55 \pm 0,01 a$ \\
\hline \multirow{6}{*}{ Fiente après 2 semaines d'élevage } & Adzopé & $2,13 \pm 0,02 b$ & $6,04 \pm 0,02 b$ \\
\hline & Agnibilékro & $2,036 \pm 0,02 b$ & $6,44 \pm 0,01 b$ \\
\hline & Azaguié & $1,97 \pm 0,02 c$ & $6,22 \pm 0,01 b$ \\
\hline & Bingerville & $2,54 \pm 0,03 a$ & $4,45 \pm 0,05 c$ \\
\hline & Dabou & $2,22 \pm 0,19 a b$ & $7,13 \pm 0,01 a$ \\
\hline & Divo & $2,45 \pm 0,02 a$ & $6,38 \pm 0,01 b$ \\
\hline \multirow{6}{*}{ Fiente après 6 mois d'élevage } & Adzopé & $11,23 \pm 0,01 \mathrm{a}$ & $9,54 \pm 0,02 a$ \\
\hline & Agnibilékro & $10,83 \pm 0,02 b$ & $8,96 \pm 0,02 b$ \\
\hline & Azaguié & $11,62 \pm 0,01 a$ & $9,44 \pm 0,01 \mathrm{a}$ \\
\hline & Bingerville & $10,75 \pm 0,02 b$ & $9,23 \pm 0,01 a$ \\
\hline & Dabou & $10,94 \pm 0,02 b$ & $8,87 \pm 0,02 b$ \\
\hline & Divo & $11,23 \pm 0,02 a$ & $8,95 \pm 0,02 b$ \\
\hline \multirow{6}{*}{$\begin{array}{l}\text { Fiente prélevée sur les marchés des différentes } \\
\text { communes d'Abidjan }\end{array}$} & Abobo gare & $8,35 \pm 0,02$ & $9,26 \pm 0,01$ \\
\hline & Adjamé marché & $8,36 \pm 0,03$ & $9,52 \pm 0,01$ \\
\hline & yopougon siporex & $8,35 \pm 0,02$ & $8,97 \pm 0,03$ \\
\hline & Cocody Angré marché cocovico & $8,35 \pm 0,02$ & $9,33 \pm 0,02$ \\
\hline & Treichville marhé & $8,35 \pm 0,02$ & $9,27 \pm 0,01$ \\
\hline & koumassi marché & $8,35 \pm 0,01$ & $9,24 \pm 0,02$ \\
\hline Fcal & & $38,1701^{* *}$ & $12,6996^{* *}$ \\
\hline
\end{tabular}




\section{Pcal} Pthéorique

0,000158

$\leq 0,01$

0,000005

$\leq 0,01$

Les moyennes affectées d'une même lettre dans une même colonne ne sont pas significativement différentes au seuil a $<0,01$ selon le test de Tukey. ns = non significative; ${ }^{*}=$ significative; ${ }^{* *}=$ hautement

significative et ${ }^{* * *}=$ très hautement significative, $\mathrm{Cal}=$ calculer, théor $=$ théorique. 
Apata et al. J. Appl. Biosci. 2014.

Évaluation des métaux traces dans les fientes de volailles sur les œufs destinés à la consommation humaine

Tableau 4: Teneurs moyennes en éléments traces métalliques considérés par la CEE $\left(\mathrm{mg} \cdot \mathrm{kg}^{-1}\right)$.

\begin{tabular}{|c|c|c|c|c|c|}
\hline \multirow[b]{2}{*}{ Type de matériel } & \multicolumn{5}{|c|}{ Eléments traces métalliques } \\
\hline & $\mathrm{Cu}$ & $\mathrm{Zn}$ & $\mathrm{Cr}$ & $\mathrm{Cd}$ & $\mathrm{Pb}$ \\
\hline copeaux de bois & 7,87 & 4,36 & 5,27 & 7.76 & 1,14 \\
\hline Aliments de poules & 21,12 & 80,37 & 9,49 & 6.05 & 10,14 \\
\hline Fiente après 2 semaines d'élevage & 9,05 & 21,76 & 7,43 & 6.11 & 2,22 \\
\hline Fiente après 6 mois d'élevage & 26,11 & 68,71 & 9,16 & 9.16 & 11,1 \\
\hline $\begin{array}{l}\text { Fiente prélevée sur les marchés } \\
\text { des différentes communes } \\
\text { d'Abidjan }\end{array}$ & 42,1 & 25,68 & 2,53 & 9.27 & 8,35 \\
\hline Valeurs limites selon la CEE & 100 & 300 & 100 & 1 & 100 \\
\hline
\end{tabular}

\section{DISCUSSION}

La présence sur les marchés de $62,65 \%$ vendeurs d'âge supérieur à 25 ans et ceux d'âge compris entre 18 et 25 ans pour une proportion de $19,64 \%$ montre que le commerce des œufs est l'affaire de personnes matures et conscientes de la fragilité des œufs, qui peuvent se casser facilement. En effet, lors de la manipulation de ceux-ci par les commerçants, il peut se produire beaucoup de perte due à une absence de concentration lors du déplacement de ces œufs, pour appliquer un traitement de nettoyage. Le nettoyage des œufs est d'autant plus nécessaire que selon la FAO (2004), les œufs souillés par des matières fécales (fientes) ou par le contenu des œufs fissurés, ainsi que les œufs cassés se dégradent plus rapidement que les œufs propres. Les résultats de notre enquête concernant le niveau intellectuel rassurent encore sur ce plan, d'autant plus que $43,45 \%$ des vendeurs ont un niveau de scolarisation du secondaire et $18,45 \%$ de niveau supérieur. Cela signifie que plus de $50 \%$ ont un niveau intellectuel à même de leur permettre de prendre conscience que les œufs peuvent se casser facilement en les manipulant, et mieux recevoir les messages de sensibilisations nécessaires pour améliorer certaines habitudes, à risques, des vendeurs de la filière volaille, notamment la manipulation des fientes de volailles sans port de gant.. En effet, la manipulation des œufs sales avec les mains nus est une source d'exposition aux éléments traces métalliques contenus dans les fientes présentes sur les coquilles d'œufs. Les résultats obtenus lors de nos travaux, ont révélés la présence de sept éléments traces métalliques essentiels et deux non essentiels, à des teneurs généralement inferieures aux valeurs limites définies par la CEE (Commission Économique des nations unies pour l'Europe) (ADEME et CHAUVIN, 2004) dans les fientes analysés. Ces valeurs limites sont dans l'ordre respectif de $200 ; 300 ; 100 ; 100$; $1 ; 50 ; 100 ; 200 \mathrm{mg}^{\mathrm{kg}} \mathrm{kg}^{-1}$ pour le fer, le zinc, le plomb, le cuivre, le cadmium, le nickel, le chrome et l'arsenic. Cependant certains éléments traces comme le fer et le cadmium ont des concentrations au dessus des valeurs seuils. Nos résultats $\mathrm{mg} \cdot \mathrm{kg}^{-1}$ sont en conformité avec ceux obtenus par Doelsch (2004) dans le cas du plomb, qui a trouvé des teneurs de l'ordre de 10 à $84.0 \mathrm{mg}^{\mathrm{kg}}{ }^{-1}$ de matière sèche de fiente de volaille. Ils sont également proches de celui obtenu par Faridullah et al (2009), avec 35,1 mg. kg-1, dans le fumier de canard. Cependant nos résultats diffèrent de Sistani et al (2003) avec 2763 mg.kg-1, dans le cas du cuivre, contre 8 à $42 \mathrm{mg} \mathrm{kg}^{-1}$ dans notre travail. Nos résultats diffèrent également ceux obtenus par Perron et Hébert en 2007 qui ont trouvé de valeurs en ETM de $2 \mathrm{mg} \cdot \mathrm{kg}^{-1}$ de matière sèche de fumier de volailles pour le plomb; 0,4 mg. $\mathrm{kg}^{-1}$ pour le cadmium; 9 mg. $\mathrm{kg}^{-1}$ pour l'arsenic contre $10 \mathrm{mg}^{\mathrm{kg}}{ }^{-1}$ pour le plomb ; 9 mg. $\mathrm{kg}^{-1}$ pour le cadmium ; 0.9 mg. $\mathrm{kg}^{-1}$ pour l'arsenic. Les fortes teneurs en fer peuvent s'expliquer par le fait que le fer est beaucoup utilisé dans les réactions métaboliques qui ont lieu dans les différents organismes vivants, (Alnaga, 2009), mais également par le fait que le fer est uniformément réparti dans toute la plante Mench et al. (1992). II est présent en grande quantité dans les végétaux, ce qui explique leur présence élevée dans les fientes de poules, qui sont principalement nourries à partir d'aliments à base de végétaux. Selon le Groupe France Agricole (2002), seule une fraction du fer absorbée via l'alimentation est utilisée. De ce fait, il paraît évident que le reste de ces éléments trace non utilises par l'organisme se retrouve dans les déchets des organismes vivants (hommes et animaux). Malgré cette présence en forte quantité dans les sédiments de fiente, le fer n'est pas considéré comme un élément toxique, car selon la législation française, la Direction Générale de l'Environnement, de la CEE, les éléments prise en compte sont le cadmium, le zinc, le plomb, le chrome, le cuivre, le mercure et le nickel (Doelsch, 2004). Ces différents 
éléments ont été trouvés en des teneurs inférieures aux valeurs seuils respectives, sauf dans le cas du cadmium, dont la valeur déterminée est de $9 \mathrm{mg} . \mathrm{kg}^{-}$ ${ }^{1}$ en moyenne contre une valeur seuil de $1 \mathrm{mg}^{\mathrm{kg}}{ }^{-1}$ : ce qui représente un risque toxicologique pour les manipulateurs des œufs, avec présence de sédiments de fientes sur la coquille. De plus la présence dans les fientes des autres éléments,

\section{CONCLUSION}

La présente étude montre que la plus part des vendeurs d'œufs de poule pondeuses sur les différents marchés de la ville d'Abidjan ont un niveau d'étude qui ne leur permet d'appréhender le risque toxicologique dû aux fientes présentes sur les coquilles de œufs qui leurs sont livrés par les fermes du secteur informel. Ces sédiments ont des charges en éléments traces métalliques en deçà des valeurs limites sauf le cadmium, dont la teneur est 9 fois plus élevée que la valeur seuil recommandé. Cela représente un réel danger pour tous ceux qui manipulent ces œufs sans protection. II est donc important qu'une campagne de sensibilisation soit menée auprès des fermes du secteur informel, afin

\section{REFERENCES BIBLIOGRAPHIQUES}

Anonyme 1, 2013. Abidjan situation géographique de la ville d'Abidjan, 1 p. http://www.technoscience.net/?onglet=glossaire\&definition $=21$ 55 site visité le 12/09/2013.

Anonyme 2, 2012. Les toxi-infection alimentaires collectives. Microbiologie 2 CAP APR, $7 p$ ADEME, 2007. Bilan des flux de contaminants entrant sur les sols agricoles de France métropolitaine $329 p$

Ademe B., Chauvin M., 2004. La réglementation française sur la valorisation agronomique des déchets organiques. Organisation et points principaux, 11p. www.ademe.fr/bretagne/.../reglementation_ DO.doc visité le 21/04/2013

Alnaga N., 2009. Synthèse de la Quinolobactine, de l'Entérobactine et leurs dérivés. Etudes de la complexation du fer. Thèse soutenue à I'Université Joseph Fourier (Grenoble 1); $213 p$

Basta N.T., Ryan A. J., Chaney L.R., 2005. Trace element chemistry in residual-treated soil: key concepts and metal bioavailability. Journal of Environmental Quality, 34 (1), pp 49-63.

Benedetto M. D., Anfossi S., Billiard E., Bonnet M., Henriot F., Kraemer F., Lechenne L., Le Herissier M., Lorin S., 1997. Méthodes considérés par la direction générale de la CEE, même avec des charges inférieures à leurs valeurs limites, représente un facteur de risque pour les vendeurs de la filière avicole. Selon Travel et al. (2012) la présence des ETM dans les fientes peut s'expliquer par le phénomène de transfert de polluants organiques persistants (POP) présents dans le sol et dans les aliments.

d'attirer leur attention sur les risques toxicologiques, dus à un contact régulier avec ces ETM, que courent les vendeurs en recevant des œufs non débarrassés des fientes présents sur les coquilles après leurs pontes. Au terme de cette étude, nous recommandons aux fermes du secteur informel de procéder à un nettoyage systématique de leurs œufs avant de les livrer sur les marchés, d'obliger au port de gants de leurs employés chargés du nettoyage. Au structure de production des aliments pour poules pondeuses, il serait important que ceux-ci fournissent aux fermes des aliments ne contenant pas de plomb, de cadmium et de mercure.

spectrométriques d'analyse et de caractérisation. Ecole des Mines de SaintEtienne, $49 \mathrm{p}$.

Boisson Y. et Teyssou R. 2002. Les toxi-infections alimentaires collectives. Revue françaises des Laboratoires, volumes 2002, Issue 348, pp 61 -66.

Boughaba R., 2012. Thèse soutenue à l'Université de Mentouri Constantine à la Faculté des Sciences de la Nature et de la Vie Département de Biologie et Ecologie, 98p

Dauguet S., Denaix L., Nguyen C., Royer E., Levasseur P., Potin-Gautier M., Lespes G.,Parat C., Héroult J., Coudure R., Chéry P., Devert M. , Robert N., Pouech P., 2011. Mesure des flux d'éléments traces $(\mathrm{Pb}, \mathrm{Cd}$, As, $\mathrm{Cu}, \mathrm{Zn}$ ) dans les sols, végétaux, porcs et lisiers des exploitations porcines du SudOuest. Innovations Agronomiques 17, 175190

Doelsch E., 2004. Eléments traces métalliques; inventaire pour l'ILE DE LA REUNION (sols, déchets, végétaux). CIRAD ; 10p.

FAO, 2008. Revue du secteur avicole Côte d'lvoire. $77 p$.

Fourmont D., 1982. Les fientes de volailles déshydratées utilisées dans l'alimentation des ruminants. Thèse de doctorat 
vétérinaire, université Claude Bernard, Lyon, $203 p$.

Faridullah, Irshad M, Yamamoto S, Honna T, Eneji AE., 2009. Characterization of trace elements in chicken and duck litter ash. Waste Management, 29(1), pp 265-271.

Faverger M. H., 2005. L'œuf, bon ou mauvais pour la santé ? Palais de la découverte ; $6 \mathrm{p}$.

Gouzy A. et Ducos G., 2008. La connaissance des éléments traces métalliques : un défi pour la gestion de l'environnement. Air Pur $N^{\circ} 75$ deuxième semestre, $\mathrm{pp}$ 6-10.

Groupe France Agricole, 2002. Guide : Engrais verts et fertilité des sols. 2ème édition; Agridecision, 305pp.

Levasseur P. et Texier C., 2001. Teneurs en éléments-traces métalliques dans les aliments et des lisiers de porcs à l'engrais, de truites et de porcelets. Journées de recherche porcine en France, 33 : pp 57-62.

Magdelaine P. et Braine A., 2008. Le marché des œufs et des ovoproduits. TeMA $n^{0} 5 ; 34 p$.

Magras C. Federighi, Soule C., 1997. Les dangers pour la santé publique liés la consommation de la viande de cheval. Rev. Sci. Tech. Off. int. Epiz., 16 (2), pp 554-563

Mench M., Juste C. et Solda P. 1992. Effets de l'utilisation de boues urbaines en essais de longue durée: accumulation des métaux par les végétaux supérieurs. Actual. Bot., 1: $p p$ 141- 156.

Perron V. et Hebert M., 2007. Caractérisation des boues d'épuration municipales. Partie II: éléments traces métalliques. Vecteur environnement ; pp 42- 46

Sistani KR, Brink GE, Mcgowen SL, Rowe De, Oldham JL., 2003. Characterization of broiler cake and broiler litter, the by-products of two management practices. Bioresource Technology, 90(1), pp 27-32.

Touré N., 2012. Évaluation de la contamination des sols, eaux et produits maraîchers par des Eléments Traces Métalliques dans la vallée du Nieky, au Sud Est de la Côte d'Ivoire. Thèse doctoral soutenue publiquement à L'Université Félix Houphouët-Boigny de Cocody (Côte d'Ivoire), 184p.

Tsen Y.H.; Hu H.H ; Lin J.S., Huang H.C.; Wang K.T., 2000. Analyse of the Salmonella typhimurium isolates from food-poisoning cause by molecular subtyping methods. Food microbiology, volume 17, Issue 2, pp $143-152$.
Travel A., Fournier A., Marchand P., Venisseau A., Le Bouquin S., Allain V., Thebault A., Mahe A., Grammont V., Badreddine R., Jurjanz S. , Feidt C., Jondreville C., 2012. Transfert de polluants organiques persistants vers l'œuf de consommation : état des lieux, modalités et facteurs de risques. Innovations Agronomiques 25, pp 313-330.

Tremel-Schaub A. et Feix I. Contamination des sols. Transfert des sols vers les plantes. Paris: EDP Sciences/ADEME, 413p. 